\title{
Minoxidil Associated Pericardial Effusion: A Case Report
}

\author{
Steven B. Deutsch ${ }^{\mathrm{a}, \mathrm{c}}$, Michael Berlowitz ${ }^{\mathrm{b}}$
}

\begin{abstract}
Minoxidil is an antihypertensive that works by directly dilating peripheral vessels. The agent produces vasodilation via a cyclic AMPmediated effect on arteriolar smooth muscle, with little effect on veins. There have been associations with its use and cause and/or exacerbation of pericardial effusions. This reported adverse effect resulted in a black box warning in the US, which states that minoxidil may cause pericarditis and pericardial effusion that may progress to tamponade. Specifically, patients with renal impairment not on dialysis and individuals with congestive heart failure are reported at higher risk. Here we report a case of a patient with underlying renal disease who presented to the hospital with hypertensive urgency. He developed a pericardial effusion soon after restarting minoxidil, which did not resolve until this medication was discontinued, despite undergoing a pericardial window.
\end{abstract}

Keywords: Pericardial effusion; Effusion; Antihypertensive; Minoxidil

\section{Introduction}

Minoxidil is an antihypertensive that works by directly dilating peripheral vessels. The agent produces vasodilation via a cyclic AMP-mediated effect on arteriolar smooth muscle, with little effect on veins. There have been associations with its use and cause and/or exacerbation of pericardial effusions [1,2]. The purpose of this study was to investigate an adverse drug event and its associated black box warning of pericardial effusion. I hypothesized that minoxidil was the cause of recurrent

Manuscript accepted for publication August 27, 2014

aUniversity of South Florida Morsani College of Medicine, Tampa, FL, USA ${ }^{b}$ College of Medicine Cardiology, Coronary Services, Cardiovascular Sciences, USF Health South Tampa Campus, Tampa, FL, USA

${ }^{\mathrm{c} C o r r e s p o n d i n g ~ A u t h o r: ~ S t e v e n ~ B . ~ D e u t s c h, ~ U n i v e r s i t y ~ o f ~ S o u t h ~ F l o r i d a ~ M o r-~}$ sani College of Medicine, 814 W San Rafael St, Tampa, FL 33629, USA. Email: sdeutsch@health.usf.edu

doi: http://dx.doi.org/10.14740/jmc1928w pericardial effusion in a patient whom underwent a pericardial window. I reviewed multiple studies consisting of observational studies, prospective observational studies using search words such as "pericardial effusion", "drug related pericardial effusion", "minoxidil and pericardial effusion", "minoxidil black box warning" in PubMed, UptoDate, and Journal of American Medical Association.

Although the cause of the pericardial effusion in the patient discussed here is uncertain, malignancy, uremia, MI and autoimmune disease were ruled out. It is important to note that despite undergoing a pericardial window, the effusion did not resolve until minoxidil was discontinued.

\section{Case Report}

A 60-year-old Caucasian male with a medical history significant for hypertension, cerebrovascular accident, abdominal aortic aneurysm, polycystic kidney disease status post right nephrectomy, sick sinus syndrome status post St. Jude pacemaker placement and diet controlled diabetes, presented to the emergency room secondary to elevated blood pressure reading greater than 200/100 at home. He reported medication compliance, except for his minoxidil, which he had not taken for 1 week. Patient also reported sharp, non-radiating chest pain exacerbated by movement. He stated that he had experienced chest pain similar in quality in the past, and that he did not believe his current pain to be cardiac in origin. Patient was admitted to the cardiac service and his minoxidil, as well as his other home medications were restarted. Blood pressure returned to baseline. A chest X-ray was obtained at the time of admission and showed enlargement of the cardiac pericardial silhouette with clear lungs and no evidence of consolidation or effusions. Brain natriuretic peptide (BNP) was found to be 987 and a two-dimensional cardiac echo was obtained which showed a moderate pericardial effusion with some signs of early tamponade physiology: diastolic right atrial collapse $(<50 \%$ of diastole $)$, mild respirophasic variation $(<25 \%$ mitral inflow variation) and dilated non-collapsible inferior vena cava (IVC), although the patient was hypertensive at the time [3]. A consult was placed to cardiothoracic surgery for consideration of a pericardial window. The following morning the pericardial window was performed and approximately $550 \mathrm{cc}$ was evacuated. Fluid was sent for cytology and cultures. Cy- 
tology was negative for malignancy and there was no growth of microorganisms. A pericardial biopsy was obtained without evidence of tumor or inflammation. Serum histone Ab, anti-DS DNA, SS-B, and SS-A were negative. C3 and C4 were within normal limits, as was myeloperoxidase $\mathrm{AB}$ and proteinase $\mathrm{AB}$. Patient was transferred to the cardiac intensive care unit for observation. He remained stable with blood pressures well controlled. He was discharged home with a refill of minoxidil.

Nine days later, the patient returned to the emergency room with reported shortness of breath, diaphoresis, and increased chest pain. Repeat two-dimensional echocardiogram was performed and demonstrated a large, free flowing pericardial effusion, circumferential to the heart with moderate atrial chamber collapse. Ejection fraction was estimated to be $55 \%$. There was evidence for increased right ventricle-left ventricle interaction demonstrated by respirophasic changes in tricuspid velocities. Features were consistent with mild tamponade physiology. A large residual pericardial effusion was identified. Cardiothoracic surgery was consulted for recurrent pericardial effusion. CT angiogram of the chest was negative for pulmonary embolus, but significant for a large pericardial effusion and small bilateral pleural effusion, right greater than left. Right thoracotomy was performed, as well as a pericardial window and biopsy. The patient's systolic blood pressure was increased by $10 \mathrm{~mm} \mathrm{Hg}$ upon drainage of the pericardial collection. Cytology was non-revealing as was cytometry. The patient's creatinine throughout the hospital course ranged from 1.0 to 2.0 with appropriate urine output and a BUN of $11-28$. Minoxidil was discontinued at that time due to high suspicion that the medication was the culprit for the recurrent pericardial effusion [4-6]. Repeat echo on post operation day 7 of pericardial window showed the estimated ejection fraction to be in the range of $55 \%$. Compared to prior transthoracic echocardiogram, there was interval resolution of large circumferential pericardial effusion. There was a small loculated inferior effusion without echocardiographic signs of tamponade.

\section{Discussion}

Minoxidil is a direct acting vasodilator used for the treatment of hypertension. Side effects observed with the drug are peripheral edema $(7 \%)$, pericardial effusion with tamponade $(3 \%)$, pericardial effusion without tamponade $(3 \%)$, angina pectoris, heart failure, pericarditis, rebound hypertension, tachycardia, and sodium and water retention. Patients with renal impairment are at higher risk for developing pericardial effusions, although this adverse effect has been documented in patients with normal renal function as well. The exact mechanism behind pericardial effusion in patients taking minoxidil is not clearly understood. Pericardial effusion in minoxidil-treated patients has been attributed to uremia, as well as the salt and water retention consequent of the vasodilatory effect of the drug. Review of the literature suggests that pericardial effusion is uncommonly observed in patients treated with minoxidil unless accompanied by cardiac or renal failure.

The patient reviewed in this case had underlying renal disease; however, renal function during hospitalization was within normal limits and at his baseline. His creatinine on discharge was 1.2, with a glomerular filtration rate of greater than 60 , despite having a diagnosis of polycystic kidney disease. Literature has hypothesized that the increased incidence of minoxidil-associated pericardial effusion is higher in the renal disease population due to their susceptibility for uremia and salt and water retention; however, that did not appear to be the explanation for our patient's adverse effect. Although the cause of the pericardial effusion in the patient discussed here is uncertain, malignancy, uremia, MI and autoimmune disease were ruled out. It is important to note that despite undergoing a pericardial window, the effusion did not resolve until minoxidil was discontinued, underlying the importance of acknowledging the link between minoxidil and pericardial effusion [7-11].

\section{References}

1. Houston MC, McChesney JA, Chatterjee K. Pericardial effusion associated with minoxidil therapy. Arch Intern Med. 1981;141(1):69-71.

2. Maisch B, Seferovic PM, Ristic AD, Erbel R, Rienmuller $\mathrm{R}$, Adler Y, Tomkowski WZ, et al. Guidelines on the diagnosis and management of pericardial diseases executive summary; The Task force on the diagnosis and management of pericardial diseases of the European society of cardiology. Eur Heart J. 2004;25(7):587-610.

3. Sagrista-Sauleda J, Merce J, Permanyer-Miralda G, Soler-Soler J. Clinical clues to the causes of large pericardial effusions. Am J Med. 2000;109(2):95-101.

4. Ben-Horin S, Bank I, Shinfeld A, Kachel E, Guetta V, Livneh A. Diagnostic value of the biochemical composition of pericardial effusions in patients undergoing pericardiocentesis. Am J Cardiol. 2007;99(9):1294-1297.

5. Levy PY, Corey R, Berger P, Habib G, Bonnet JL, Levy S, Messana T, et al. Etiologic diagnosis of 204 pericardial effusions. Medicine (Baltimore). 2003;82(6):385-391.

6. Corey GR, Campbell PT, Van Trigt P, Kenney RT, O'Connor CM, Sheikh KH, Kisslo JA, et al. Etiology of large pericardial effusions. Am J Med. 1993;95(2):209213.

7. Franciosa JA, Jordan RA, Wilen MM, Leddy CL. Minoxidil in patients with chronic left heart failure: contrasting hemodynamic and clinical effects in a controlled trial. Circulation. 1984;70(1):63-68.

8. Go AS, Bauman MA, Coleman King SM, Fonarow GC, Lawrence W, Williams KA, Sanchez E. An effective approach to high blood pressure control: a science advisory from the American Heart Association, the American College of Cardiology, and the Centers for Disease Control and Prevention. Hypertension. 2014;63(4):878-885.

9. James PA, Oparil S, Carter BL, Cushman WC, Dennison-Himmelfarb C, Handler J, Lackland DT, et al. 2014 evidence-based guideline for the management of high blood pressure in adults: report from the panel members appointed to the Eighth Joint National Committee (JNC 8). JAMA. 2014;311(5):507-520.

10. The fourth report on the diagnosis, evaluation, and treat- 
ment of high blood pressure in children and adolescents. Pediatrics. 2004;114(2 Suppl 4th Report):555-576.

11. Weber MA, Schiffrin EL, White WB, Mann S, Lindholm LH, Kenerson JG, Flack JM, et al. Clinical prac- tice guidelines for the management of hypertension in the community: a statement by the American Society of Hypertension and the International Society of Hypertension. J Clin Hypertens (Greenwich). 2014;16(1):14-26. 